\title{
Strong convergence of a composite Halpern-type iteration for a family of nonexpansive mappings in $\mathrm{CAT}(0)$ spaces
}

\author{
Sajad Ranjbar
}

\begin{abstract}
In this paper, we prove the strong convergence of the composite Halpern-type iteration for a family of nonexpansive mappings in CAT(0) spaces and compare our results with several recent results in this subject. Also, the inexact version of the Halpern iteration is studied in CAT $(0)$ spaces.
\end{abstract}

\section{Introduction and Preliminaries}

Let $C$ be a nonempty subset of a metric space $(X, d) . T: C \rightarrow C$ is called nonexpansive if for each $x, y \in C, d(T x, T y) \leq d(x, y)$. A point $x \in C$ is called a fixed point of $T$ if $T x=x$. We denote $F(T):=\{x \in C: T(x)=x\}$.

Halpern [9] proved the strong convergence of the iteration

$$
x_{n+1}=\alpha_{n} u+\left(1-\alpha_{n}\right) T x_{n},
$$

where $u \in C$ is an arbitrary (but fixed) element in $C$, under suitable assumptions on the control sequence $\alpha_{n}$. Also, he showed that the assumptions $C 1: \lim _{n \rightarrow \infty} \alpha_{n}=0$, $C 2: \sum_{n=1}^{\infty} \alpha_{n}=\infty$,

Key Words: Fixed point, Strong convergence, Composite Halpern-type iteration, Nonexpansive mapping, $\mathrm{CAT}(0)$ space.

2010 Mathematics Subject Classification: Primary 47H10, 47H09; Secondary 26A18.

Received: 01.03.2017

Revised: 21.03.2017

Accepted: 31.03.2017 
STRONG CONVERGENCE OF A COMPOSITE HALPERN TYPE ITERATION FOR A FAMILY OF NONEXPANSIVE MAPPINGS IN CAT(0) SPACES

are necessary for the strong convergence of the iteration (1.1) to a fixed point of $T$. He also proposed the following open problem:

Are conditions $C 1$ and $C 2$ sufficient for the strong convergence of the sequence generated by (1.1) to a fixed point of $T$ ?

Lions [14] proved the strong convergence of the Halpern iteration under the conditions $C 1, C 2$ and $(C 3) \lim _{n \rightarrow \infty} \frac{\alpha_{n+1}-\alpha_{n}}{\alpha_{n+1}^{2}}=0$.

Wittmann [20] improved the result of Lions under the assumptions $(C 1),(C 2)$ and $(C 4) \sum_{n=1}^{\infty}\left|\alpha_{n+1}-\alpha_{n}\right|<\infty$.

An analogue of Wittmann's result was first proved by Reich [16] for Banach spaces with a weakly sequentially continuous duality mapping. Shioji and Takahashi [18] extended Wittmann's result to Banach spaces with uniformly Gateaux differentiable norm. Xu [21, 22] proved the strong convergence of the Halpern iteration provided that the conditions (C1), (C2) and $(C 5) \lim _{n \rightarrow \infty} \frac{\alpha_{n}}{\alpha_{n+1}}=1$ are satisfied.

Suzuki [19] and Chidume-Chidume [5], independently, proved that the conditions $(C 1)$ and $(C 2)$ are sufficient for the strong convergence of the following iterative sequence:

$$
x_{n+1}=\alpha_{n} u+\left(1-\alpha_{n}\right)\left(\lambda x_{n}+(1-\lambda) T x_{n}\right),
$$

to a fixed point of $T$, where $T$ is a nonexpansive mapping on a closed and convex subset $C$ of a Banach space with uniformly Gateaux differentiable norm and $x_{1}, u \in C$. Therefore Halpern open problem has a positive solution when the nonexpansive operator is a convex combination of the identity operator and another nonexpansive operator. Qin, Su and Shang [15] introduced the composite iteration scheme as follows:

$$
\left\{\begin{array}{l}
z_{n}=\gamma_{n} x_{n}+\left(1-\gamma_{n}\right) T x_{n} \\
y_{n}=\beta_{n} x_{n}+\left(1-\beta_{n}\right) T z_{n} \\
x_{n+1}=\alpha_{n} u+\left(1-\alpha_{n}\right) y_{n}
\end{array}\right.
$$

where $T$ is a nonexpansive mapping on a closed and convex subset $C$ of a uniformly smooth Banach space, $F(T) \neq \emptyset, u \in C$ is an arbitrary (but fixed) element in $C$, and $\left\{\alpha_{n}\right\},\left\{\beta_{n}\right\}$ and $\left\{\gamma_{n}\right\}$ are sequences in $[0,1]$. They proved the sequence $\left\{x_{n}\right\}$ defined by (1.3) converges strongly to a fixed point of $T$ under appropriate assumptions on the sequences $\left\{\alpha_{n}\right\},\left\{\beta_{n}\right\}$ and $\left\{\gamma_{n}\right\}$.

The main goal of this paper is to prove the strong convergence of the sequence generated by (1.3) for a family of nonexpansive mappings in CAT(0) spaces. In the sequel, we introduce $\operatorname{CAT}(0)$ spaces. Let $(X, d)$ be a metric space and $x, y \in X$. A geodesic path joining $x$ to $y$ is an isometry $c:[0, d(x, y)] \longrightarrow X$ such that $c(0)=x, c(d(x, y))=y$. The image of a geodesic path joining $x$ to $y$ is called a geodesic segment between $x$ and $y$. When it is unique, this geodesic 
STRONG CONVERGENCE OF A COMPOSITE HALPERN TYPE ITERATION

segment is denoted by $[x, y]$. The space $(X, d)$ is said to be a geodesic space if every two points of $X$ are joined by a geodesic, and $X$ is said to be uniquely geodesic if there is exactly one geodesic joining $x$ and $y$ for each $x, y \in X$. A subset $Y \subseteq X$ is said to be convex if $Y$ includes every geodesic segment joining any two of its points.

A geodesic triangle $\triangle\left(x_{1}, x_{2}, x_{3}\right)$ in a geodesic space $X$ consists of three points $x_{1}, x_{2}, x_{3}$ in $X$ (the vertices of $\triangle$ ) and a geodesic segment between each pair of vertices. A comparison triangle for the geodesic triangle $\triangle\left(x_{1}, x_{2}, x_{3}\right)$ is the triangle $\bar{\triangle}\left(\overline{x_{1}}, \overline{x_{2}}, \overline{x_{3}}\right)$ in the Euclidean plane $\mathbb{R}^{2}$ such that

$$
d_{\mathbb{R}^{2}}\left(\overline{x_{i}}, \overline{x_{j}}\right)=d\left(x_{i}, x_{j}\right) \text { for } i, j \in\{1,2,3\} .
$$

A geodesic space $X$ is a $\operatorname{CAT}(0)$ space if for each geodesic triangle $\triangle$ in $X$ and its comparison triangle $\bar{\triangle}$ in $\mathbb{R}^{2}$, the $\mathrm{CAT}(0)$ inequality

$$
d(x, y) \leq d_{\mathbb{R}^{2}}(\bar{x}, \bar{y})
$$

is satisfied for all $x, y \in \triangle$ and $\bar{x}, \bar{y} \in \bar{\triangle}$. It is well-known that a $\operatorname{CAT}(0)$ space is an uniquely geodesic space.

There are several examples of $\mathrm{CAT}(0)$ spaces among metric structures. For example, any complete, simply connected Riemannian manifold having nonpositive sectional curvature is a CAT(0) space. Other examples include preHilbert spaces (see [2]), $\mathbb{R}$-trees (see [11]), Euclidean buildings (see [3]), the complex Hilbert ball with a hyperbolic metric (see [8]), and many others. For a thorough discussion of these spaces and their fundamental role in geometry, we refer the reader to Bridson and Haefliger [2].

Fixed-point theory in CAT(0) spaces was first studied by Kirk (see [12, 13]). He showed that every nonexpansive (single-valued) mapping defined on a bounded, closed and convex subset of a complete CAT(0) space always has a fixed point. Since then, the fixed-point theory for single-valued and multivalued mappings in $\mathrm{CAT}(0)$ spaces has been rapidly developed, and many papers have appeared. It is worth mentioning that fixed-point theorems in CAT(0) spaces (specially in $\mathbb{R}$-trees) can be applied to graph theory, biology, and computer science.

In this article, we write $(1-t) x \oplus t y$ for the unique point $z$ in the geodesic segment joining $x$ to $y$ such that $d(z, x)=t d(x, y), d(z, y)=(1-t) d(x, y)$. If $X$ is a $\operatorname{CAT}(0)$ space and $x, y \in X$, then $[x, y]:=\{(1-t) x \oplus t y: t \in[0,1]\}$ and a subset $C$ of $X$ is convex if $[x, y] \subseteq C$, for all $x, y \in C$.

Saejung [17] proved the strong convergence of Halpern's iteration in CAT $(0)$ spaces. He considered the iteration:

$$
x_{n+1}=\alpha_{n} u \oplus\left(1-\alpha_{n}\right) T_{n} x_{n},
$$


STRONG CONVERGENCE OF A COMPOSITE HALPERN TYPE ITERATION

where $\left\{T_{n}\right\}$ is a family of nonexpansive mappings on closed and convex subset $C$ of a $\operatorname{CAT}(0)$ space with $\bigcap_{n=1}^{\infty} F\left(T_{n}\right) \neq \emptyset$ and $x_{1}, u \in C$. He proved the strong convergence of this iteration to a common fixed point of $\left\{T_{n}\right\}$, under the conditions $C 1, C 2$ and $C 4$ or $C 5$ and an appropriate condition on $\left\{T_{n}\right\}$ which is introduced in (3.1).

In this paper, we prove the strong convergence of the sequence generated by

$$
\left\{\begin{array}{l}
z_{n}=\gamma_{n} x_{n} \oplus\left(1-\gamma_{n}\right) T_{n} x_{n} \\
y_{n}=\beta_{n} x_{n} \oplus\left(1-\beta_{n}\right) T_{n} z_{n} \\
x_{n+1}=\alpha_{n} u \oplus\left(1-\alpha_{n}\right) y_{n}
\end{array}\right.
$$

where $\left\{T_{n}\right\}$ is a family of nonexpansive mappings on closed and convex subset $C$ of a complete $\mathrm{CAT}(0)$ space $X$ with $\bigcap_{n=1}^{\infty} F\left(T_{n}\right) \neq \emptyset, x_{1}, u \in C$ and $\left\{\alpha_{n}\right\}$, $\left\{\beta_{n}\right\},\left\{\gamma_{n}\right\}$ are sequences in $[0,1]$ that satisfy the following conditions:

$\left(A_{1}\right) \sum_{n=1}^{\infty} \alpha_{n}=\infty$

$\left(A_{2}\right) \alpha_{n} \rightarrow 0, \beta_{n} \rightarrow 0, \liminf _{n} \gamma_{n}>0$

$\left(A_{3}\right) \sum_{n=1}^{\infty}\left|\alpha_{n+1}-\alpha_{n}\right|<\infty$ or $\lim _{n \rightarrow \infty} \frac{\alpha_{n}}{\alpha_{n+1}}=1$;

$\left(A_{4}\right) \sum_{n=1}^{\infty}\left|\beta_{n+1}-\beta_{n}\right|<\infty$ or $\lim _{n \rightarrow \infty} \frac{\left|\beta_{n+1}-\beta_{n}\right|}{\alpha_{n+1}}=0$;

$\left(A_{5}\right) \sum_{n=1}^{\infty}\left|\gamma_{n+1}-\gamma_{n}\right|<\infty$ or $\lim _{n \rightarrow \infty} \frac{\left|\gamma_{n+1}-\gamma_{n}\right|}{\alpha_{n+1}}=0$.

This result extends the result of [15] in CAT(0) spaces. (1.5) is also a modified Ishikawa iteration. If $\beta_{n} \equiv 0$ and $\gamma_{n} \equiv 1$ in (1.5), then we get (1.4) which has been considered by Saejung [17].

On the other hand, if $\gamma_{n} \equiv 1$, then (1.5) can be viewed as a modified Halpern iteration

$$
\left\{\begin{array}{l}
y_{n}=\beta_{n} x_{n} \oplus\left(1-\beta_{n}\right) T_{n} x_{n} \\
x_{n+1}=\alpha_{n} u \oplus\left(1-\alpha_{n}\right) y_{n} .
\end{array}\right.
$$

In (1.6), if $T_{n} \equiv T$ then (1.6) has been considered by Cuntavepanit and Panyanak [6] in $\mathrm{CAT}(0)$ spaces and by Kim and $\mathrm{Xu}$ [10] in Banach spaces.

\section{Some Lemmas}

The following lemmas are needed in the sequel.

Lemma 2.1. [7] Let $(X, d)$ be a $C A T(0)$ space. Then, for all $x, y, z \in X$ and all $t \in[0,1]:$

(1) $\quad d^{2}(t x \oplus(1-t) y, z) \leq t d^{2}(x, z)+(1-t) d^{2}(y, z)-t(1-t) d^{2}(x, y)$,

(2) $d(t x \oplus(1-t) y, z) \leq t d(x, z)+(1-t) d(y, z)$,

In addition, by (1), we have

$$
d(t x \oplus(1-t) y, t x \oplus(1-t) z) \leq(1-t) d(y, z) .
$$


Lemma 2.2. [4] If $(X, d)$ is a $C A T(0)$ space. Then, for all $x, y \in X$ and all $t, s \in[0,1]$, we have

$$
d(t x \oplus(1-t) y, s x \oplus(1-s) y)=|t-s| d(x, y) .
$$

Recall that a Banach limit is a continuous linear functional $\mu$ on $l^{\infty}$ such that $\|\mu\|=\mu(1,1, \ldots)=1$ and $\mu_{n}\left(a_{n}\right)=\mu_{n}\left(a_{n+1}\right)$, for all $\left\{a_{n}\right\} \in l^{\infty}$.

Lemma 2.3. [18] Let $\left(a_{1}, a_{2}, \ldots\right)$ be in $l^{\infty}$ such that $\mu_{n}\left(a_{n}\right) \leq 0$ for all Banach limits $\mu$ and $\limsup _{n}\left(a_{n+1}-a_{n}\right) \leq 0$, then $\limsup _{n} a_{n} \leq 0$.

Lemma 2.4. [1] Let $\left\{s_{n}\right\}$ be a sequence of nonnegative real numbers, $\left\{\alpha_{n}\right\}$ a sequence of real numbers in $[0,1]$ with $\sum_{n=1}^{\infty} \alpha_{n}=\infty,\left\{u_{n}\right\}$ a sequence of nonnegative real numbers with $\sum_{n=1}^{\infty} u_{n}<\infty$, and $\left\{t_{n}\right\}$ a sequence of real numbers with limsup $t_{n} \leq 0$. Suppose that

$$
s_{n+1} \leq\left(1-\alpha_{n}\right) s_{n}+\alpha_{n} t_{n}+u_{n},
$$

for all $n \in \mathbb{N}$. Then $\lim _{n \rightarrow \infty} s_{n}=0$.

Lemma 2.5. [17] Let $C$ be a closed and convex subset of a complete CAT(0) space $X$ and let $T: C \longrightarrow C$ be a nonexpansive mapping. Let $u \in C$ be fixed. For each $t \in(0,1)$, the mapping $S_{t}: C \longrightarrow C$ defined by

$$
S_{t} x=t u \oplus(1-t) T x, \quad \forall x \in C,
$$

has a unique fixed point $x_{t} \in C$, that is,

$$
x_{t}=S_{t} x_{t}=t u \oplus(1-t) T x_{t} .
$$

Lemma 2.6. [17] Let $C$ be a closed and convex subset of a complete $C A T(0)$ space $X$ and let $T: C \longrightarrow C$ be a nonexpansive mapping. Then $F(T) \neq \emptyset$ if and only if $\left\{x_{t}\right\}$ given by the formula (2.1) remains bounded as $t \rightarrow 0$. In this case, the following statements hold:

(1) $\left\{x_{t}\right\}$ converges to the unique fixed point $z$ of $T$, which is the nearest point of $F(T)$ to u;

$(2) d^{2}(u, z) \leq \mu_{n} d^{2}\left(u, x_{n}\right)$, for all Banach limits $\mu$ and all bounded sequences $\left\{x_{n}\right\}$ with $d\left(x_{n}, T x_{n}\right) \rightarrow 0$.

\section{Main Results}

In the setting of the CAT(0) spaces, we prove the strong convergence of the composite Halpern-type iteration generated by (1.5) and compare our results with other results by some examples. Also, the inexact version of the Halpern iteration is studied. 
STRONG CONVERGENCE OF A COMPOSITE HALPERN TYPE ITERATION

\subsection{Composite Halpern Iteration}

The following concept was introduced by Aoyama et al. [1]. Let $C$ be a subset of a metric space $X$ and $\left\{T_{n}\right\}_{n=1}^{\infty}: C \rightarrow C$ is a countable family of mappings. The family $\left\{T_{n}\right\}$ satisfies AKTT-condition if

$$
\sum_{n=1}^{\infty} \sup \left\{d\left(T_{n+1} z, T_{n} z\right): \quad z \in B\right\}<\infty,
$$

for each bounded subset $B$ of $C$.

If $C$ is a closed subset of $X$ and $\left\{T_{n}\right\}$ satisfies AKTT-condition, then we can define $T: C \rightarrow C$ such that

$$
T x=\lim _{n \rightarrow \infty} T_{n} x, \quad(x \in C) .
$$

In this case, we also say $\left(T_{n}, T\right)$ satisfies AKTT-condition.

The following theorem extends Theorem 3.2 of Saejung [17].

Theorem 3.1. Let $C$ be a closed and convex subset of complete $C A T(0)$ space $X$ and let $\left\{T_{n}\right\}_{n=1}^{\infty}$ be a family of nonexpansive mappings of $C$ to itself such that

$$
\left\{\begin{array}{l}
(A)\left(T_{n}, T\right) \text { satisfies } A K T T \text { - condition, } \\
(B) F(T)=\bigcap_{n=1}^{\infty} F\left(T_{n}\right) \neq \emptyset .
\end{array}\right.
$$

Given a point $u \in C$ and the initial guess $x_{0} \in C$ is chosen arbitrarily. If $\left\{x_{n}\right\}$ is composite process generated by (1.5), where $\left\{\alpha_{n}\right\},\left\{\beta_{n}\right\}$ and $\left\{\gamma_{n}\right\}$ in $[0,1]$ satisfy $A_{1}-A_{5}$ then $\left\{x_{n}\right\}$ converges strongly to $z \in F(T)=\bigcap_{n=1}^{\infty} F\left(T_{n}\right)$, which is the nearest point of $F(T)$ to $u$.

Proof. Let $p \in \bigcap_{n=1}^{\infty} F\left(T_{n}\right)$. We have

$$
\begin{aligned}
d\left(z_{n}, p\right) & \leq \gamma_{n} d\left(x_{n}, p\right)+\left(1-\gamma_{n}\right) d\left(T_{n} x_{n}, p\right) \\
& \leq d\left(x_{n}, p\right),
\end{aligned}
$$

which implies

$$
\begin{aligned}
d\left(y_{n}, p\right) & \leq \beta_{n} d\left(x_{n}, p\right)+\left(1-\beta_{n}\right) d\left(T_{n} z_{n}, p\right) \\
& \leq \beta_{n} d\left(x_{n}, p\right)+\left(1-\beta_{n}\right) d\left(z_{n}, p\right) \\
& \leq d\left(x_{n}, p\right) .
\end{aligned}
$$


This follows that

$$
\begin{aligned}
d\left(x_{n+1}, p\right) & \leq \alpha_{n} d(u, p)+\left(1-\alpha_{n}\right) d\left(y_{n}, p\right) \\
& \leq \alpha_{n} d(u, p)+\left(1-\alpha_{n}\right) d\left(x_{n}, p\right) \\
& \leq \max \left\{d(u, p), d\left(x_{n}, p\right)\right\} \\
& \leq \ldots \leq \max \left\{d(u, p), d\left(x_{0}, p\right)\right\} .
\end{aligned}
$$

Thus $\left\{x_{n}\right\},\left\{y_{n}\right\},\left\{z_{n}\right\},\left\{T_{n} x_{n}\right\}$ and $\left\{T_{n} z_{n}\right\}$ are bounded. In the sequel, we show $d\left(x_{n+1}, x_{n}\right) \rightarrow 0$. We have

$$
\begin{aligned}
& d\left(z_{n}, z_{n-1}\right)=d\left(\gamma_{n} x_{n} \oplus\left(1-\gamma_{n}\right) T_{n} x_{n}, \gamma_{n-1} x_{n-1} \oplus\left(1-\gamma_{n-1}\right) T_{n-1} x_{n-1}\right) \\
& \leq d\left(\gamma_{n} x_{n} \oplus\left(1-\gamma_{n}\right) T_{n} x_{n}, \gamma_{n} x_{n} \oplus\left(1-\gamma_{n}\right) T_{n} x_{n-1}\right) \\
&+d\left(\gamma_{n} x_{n} \oplus\left(1-\gamma_{n}\right) T_{n} x_{n-1}, \gamma_{n} x_{n} \oplus\left(1-\gamma_{n}\right) T_{n-1} x_{n-1}\right) \\
&+d\left(\gamma_{n} x_{n} \oplus\left(1-\gamma_{n}\right) T_{n-1} x_{n-1}, \gamma_{n-1} x_{n} \oplus\left(1-\gamma_{n-1}\right) T_{n-1} x_{n-1}\right) \\
&+d\left(\gamma_{n-1} x_{n} \oplus\left(1-\gamma_{n-1}\right) T_{n-1} x_{n-1}, \gamma_{n-1} x_{n-1} \oplus\left(1-\gamma_{n-1}\right) T_{n-1} x_{n-1}\right) \\
& \leq\left(1-\gamma_{n}\right) d\left(T_{n} x_{n}, T_{n} x_{n-1}\right)+\left(1-\gamma_{n}\right) d\left(T_{n} x_{n-1}, T_{n-1} x_{n-1}\right) \\
&+\left|\gamma_{n}-\gamma_{n-1}\right| d\left(x_{n}, T_{n-1} x_{n-1}\right)+\gamma_{n-1} d\left(x_{n}, x_{n-1}\right) \\
& \leq\left(1-\gamma_{n}\right) d\left(x_{n}, x_{n-1}\right)+d\left(T_{n} x_{n-1}, T_{n-1} x_{n-1}\right) \\
&+\left|\gamma_{n}-\gamma_{n-1}\right| d\left(x_{n}, T_{n-1} x_{n-1}\right)+\gamma_{n-1} d\left(x_{n}, x_{n-1}\right) .
\end{aligned}
$$

With a similar computation, we get

$$
\begin{aligned}
d\left(y_{n}, y_{n-1}\right) \leq & \left(1-\beta_{n}\right) d\left(z_{n}, z_{n-1}\right)+d\left(T_{n} z_{n-1}, T_{n-1} z_{n-1}\right) \\
& +\left|\beta_{n}-\beta_{n-1}\right| d\left(x_{n}, T_{n-1} z_{n-1}\right)+\beta_{n-1} d\left(x_{n}, x_{n-1}\right) .
\end{aligned}
$$

Therefore

$$
\begin{aligned}
& d\left(x_{n+1}, x_{n}\right)=d\left(\alpha_{n} u \oplus\left(1-\alpha_{n}\right) y_{n}, \alpha_{n-1} u \oplus\left(1-\alpha_{n-1}\right) y_{n-1}\right) \\
& \leq d\left(\alpha_{n} u \oplus\left(1-\alpha_{n}\right) y_{n}, \alpha_{n} u \oplus\left(1-\alpha_{n}\right) y_{n-1}\right) \\
& \quad+d\left(\alpha_{n} u \oplus\left(1-\alpha_{n}\right) y_{n-1}, \alpha_{n-1} u \oplus\left(1-\alpha_{n-1}\right) y_{n-1}\right) \\
& \leq\left(1-\alpha_{n}\right) d\left(y_{n}, y_{n-1}\right)+\left|\alpha_{n}-\alpha_{n-1}\right| d\left(u, y_{n-1}\right) \\
& \leq\left(1-\alpha_{n}\right)\left(\left(1-\beta_{n}\right) d\left(z_{n}, z_{n-1}\right)+d\left(T_{n} z_{n-1}, T_{n-1} z_{n-1}\right)\right. \\
& \quad\left.+\left|\beta_{n}-\beta_{n-1}\right| d\left(x_{n}, T_{n-1} z_{n-1}\right)+\beta_{n-1} d\left(x_{n}, x_{n-1}\right)\right)+\left|\alpha_{n}-\alpha_{n-1}\right| d\left(u, y_{n-1}\right)
\end{aligned}
$$




$$
\begin{aligned}
& \leq\left(1-\alpha_{n}\right)\left(( 1 - \beta _ { n } ) \left(\left(1-\gamma_{n}\right) d\left(x_{n}, x_{n-1}\right)+d\left(T_{n} x_{n-1}, T_{n-1} x_{n-1}\right)\right.\right. \\
& \left.+\left|\gamma_{n}-\gamma_{n-1}\right| d\left(x_{n}, T_{n-1} x_{n-1}\right)+\gamma_{n-1} d\left(x_{n}, x_{n-1}\right)\right)+d\left(T_{n} z_{n-1}, T_{n-1} z_{n-1}\right) \\
& \left.+\left|\beta_{n}-\beta_{n-1}\right| d\left(x_{n}, T_{n-1} z_{n-1}\right)+\beta_{n-1} d\left(x_{n}, x_{n-1}\right)\right)+\left|\alpha_{n}-\alpha_{n-1}\right| d\left(u, y_{n-1}\right) \\
& =\left(1-\alpha_{n}\right)\left(1-\beta_{n}\right)\left(1-\gamma_{n}\right) d\left(x_{n}, x_{n-1}\right)+\left(1-\alpha_{n}\right)\left(1-\beta_{n}\right) d\left(T_{n} x_{n-1}, T_{n-1} x_{n-1}\right) \\
& +\left(1-\alpha_{n}\right)\left(1-\beta_{n}\right)\left|\gamma_{n}-\gamma_{n-1}\right| d\left(x_{n}, T_{n-1} x_{n-1}\right)+\left(1-\alpha_{n}\right)\left(1-\beta_{n}\right) \gamma_{n-1} d\left(x_{n}, x_{n-1}\right) \\
& +\left(1-\alpha_{n}\right) d\left(T_{n} z_{n-1}, T_{n-1} z_{n-1}\right)+\left(1-\alpha_{n}\right)\left|\beta_{n}-\beta_{n-1}\right| d\left(x_{n}, T_{n-1} z_{n-1}\right) \\
& +\left(1-\alpha_{n}\right) \beta_{n-1} d\left(x_{n}, x_{n-1}\right)+\left|\alpha_{n}-\alpha_{n-1}\right| d\left(u, y_{n-1}\right) \\
& \leq\left(\left(1-\alpha_{n}\right)\left(1-\beta_{n}\right)\left(1-\gamma_{n}\right)+\left(1-\alpha_{n}\right)\left(1-\beta_{n}\right) \gamma_{n-1}\right. \\
& \left.+\left(1-\alpha_{n}\right) \beta_{n-1}\right) d\left(x_{n}, x_{n-1}\right)+\left|\gamma_{n}-\gamma_{n-1}\right| d\left(x_{n}, T_{n-1} x_{n-1}\right) \\
& +\left|\beta_{n}-\beta_{n-1}\right| d\left(x_{n}, T_{n-1} z_{n-1}\right)+\left|\alpha_{n}-\alpha_{n-1}\right| d\left(u, y_{n-1}\right) \\
& +d\left(T_{n} x_{n-1}, T_{n-1} x_{n-1}\right)+d\left(T_{n} z_{n-1}, T_{n-1} z_{n-1}\right) \\
& =\left(1-\alpha_{n}\right)\left(1+\gamma_{n-1}-\gamma_{n}+\beta_{n-1}-\beta_{n}+\left(\gamma_{n}-\gamma_{n-1}\right) \beta_{n}\right) d\left(x_{n}, x_{n-1}\right) \\
& +\left|\gamma_{n}-\gamma_{n-1}\right| d\left(x_{n}, T_{n-1} x_{n-1}\right)+\left|\beta_{n}-\beta_{n-1}\right| d\left(x_{n}, T_{n-1} z_{n-1}\right) \\
& +\left|\alpha_{n}-\alpha_{n-1}\right| d\left(u, y_{n-1}\right)+d\left(T_{n} x_{n-1}, T_{n-1} x_{n-1}\right)+d\left(T_{n} z_{n-1}, T_{n-1} z_{n-1}\right) \\
& \leq\left(1-\alpha_{n}\right)\left(1+\left|\gamma_{n-1}-\gamma_{n}\right|+\left|\beta_{n-1}-\beta_{n}\right|+\left|\gamma_{n}-\gamma_{n-1}\right| \beta_{n}\right) d\left(x_{n}, x_{n-1}\right) \\
& +\left|\gamma_{n}-\gamma_{n-1}\right| d\left(x_{n}, T_{n-1} x_{n-1}\right)+\left|\beta_{n}-\beta_{n-1}\right| d\left(x_{n}, T_{n-1} z_{n-1}\right) \\
& +\left|\alpha_{n}-\alpha_{n-1}\right| d\left(u, y_{n-1}\right)+d\left(T_{n} x_{n-1}, T_{n-1} x_{n-1}\right)+d\left(T_{n} z_{n-1}, T_{n-1} z_{n-1}\right) \\
& \leq\left(1-\alpha_{n}\right) d\left(x_{n}, x_{n-1}\right)+\left(2\left|\gamma_{n-1}-\gamma_{n}\right|+\left|\beta_{n-1}-\beta_{n}\right|\right) d\left(x_{n}, x_{n-1}\right) \\
& +\left|\gamma_{n}-\gamma_{n-1}\right| d\left(x_{n}, T_{n-1} x_{n-1}\right)+\left|\beta_{n}-\beta_{n-1}\right| d\left(x_{n}, T_{n-1} z_{n-1}\right) \\
& +\left|\alpha_{n}-\alpha_{n-1}\right| d\left(u, y_{n-1}\right)+d\left(T_{n} x_{n-1}, T_{n-1} x_{n-1}\right)+d\left(T_{n} z_{n-1}, T_{n-1} z_{n-1}\right) \text {. }
\end{aligned}
$$

Hence

$$
\begin{aligned}
d\left(x_{n+1}, x_{n}\right) \leq & \left(1-\alpha_{n}\right) d\left(x_{n}, x_{n-1}\right) \\
& +M\left(3\left|\gamma_{n}-\gamma_{n-1}\right|+2\left|\beta_{n}-\beta_{n-1}\right|+\left|\alpha_{n}-\alpha_{n-1}\right|\right) \\
& +\sup \left\{d\left(T_{n} u, T_{n-1} u\right): u \in\left\{x_{n}\right\}\right\}+\sup \left\{d\left(T_{n} u, T_{n-1} u\right): u \in\left\{z_{n}\right\}\right\},
\end{aligned}
$$

where $M \geq \max \left\{d\left(x_{n}, x_{n-1}\right), d\left(x_{n}, T_{n-1} x_{n-1}\right), d\left(x_{n}, T_{n-1} z_{n-1}\right), d\left(u, y_{n-1}\right)\right\}$. Now, by Lemma 2.4, the conditions $A_{1}, A_{3}, A_{4}, A_{5}$ and the condition $A$ of (3.3) we obtain

$$
d\left(x_{n+1}, x_{n}\right) \rightarrow 0
$$

On the other hand

$$
\begin{aligned}
d\left(x_{n}, T_{n} x_{n}\right) & \leq d\left(x_{n}, x_{n+1}\right)+d\left(x_{n+1}, y_{n}\right)+d\left(y_{n}, T_{n} z_{n}\right)+d\left(T_{n} z_{n}, T_{n} x_{n}\right) \\
& \leq d\left(x_{n}, x_{n+1}\right)+\alpha_{n} d\left(u, y_{n}\right)+\beta_{n} d\left(x_{n}, T_{n} z_{n}\right)+d\left(z_{n}, x_{n}\right) \\
& =d\left(x_{n}, x_{n+1}\right)+\alpha_{n} d\left(u, y_{n}\right)+\beta_{n} d\left(x_{n}, T_{n} z_{n}\right)+\left(1-\gamma_{n}\right) d\left(x_{n}, T_{n} x_{n}\right),
\end{aligned}
$$


which implies

$$
\gamma_{n} d\left(x_{n}, T_{n} x_{n}\right) \leq d\left(x_{n}, x_{n+1}\right)+\alpha_{n} d\left(u, y_{n}\right)+\beta_{n} d\left(x_{n}, T_{n} z_{n}\right) .
$$

This together with (3.4) follow $\lim _{n \rightarrow \infty} \gamma_{n} d\left(x_{n}, T_{n} x_{n}\right)=0$, which by $\lim _{\inf } \gamma_{n}>$ 0 implies

$$
d\left(x_{n}, T_{n} x_{n}\right) \rightarrow 0
$$

Also, we have

$$
\begin{aligned}
d\left(x_{n}, T x_{n}\right) & \leq d\left(x_{n}, T_{n} x_{n}\right)+d\left(T_{n} x_{n}, T x_{n}\right) \\
& \leq d\left(x_{n}, T_{n} x_{n}\right)+\sup \left\{d\left(T_{n} y, T y\right): y \in\left\{x_{n}\right\}\right\} \\
& \leq d\left(x_{n}, T_{n} x_{n}\right)+\sup \left\{\sum_{k=n}^{\infty} d\left(T_{k} y, T_{k+1} y\right): y \in\left\{x_{n}\right\}\right\} \\
& \leq d\left(x_{n}, T_{n} x_{n}\right)+\sum_{k=n}^{\infty} \sup \left\{d\left(T_{k} y, T_{k+1} y\right): y \in\left\{x_{n}\right\}\right\}
\end{aligned}
$$

which together with (3.5) and AKKT-condition imply

$$
d\left(x_{n}, T x_{n}\right) \rightarrow 0 .
$$

Now, define $S_{t} x=t u \oplus(1-t) T x$, for all $x \in C$. By Lemma 2.5, for all $t \in[0,1]$, we suppose $x_{t}$ is the unique fixed point of $S_{t}$. By Lemma 2.6, $\lim _{t \rightarrow 0} x_{t}=p$, where $p \in F(T)$ and $p$ is the nearest point of $F(T)$ to $u$. In the sequel, we show $x_{n} \rightarrow p$. We have

$$
\begin{aligned}
d^{2}\left(x_{n+1}, p\right)= & d^{2}\left(\alpha_{n} u \oplus\left(1-\alpha_{n}\right) y_{n}, p\right) \\
\leq & \alpha_{n} d^{2}(u, p)+\left(1-\alpha_{n}\right) d^{2}\left(y_{n}, p\right)-\alpha_{n}\left(1-\alpha_{n}\right) d^{2}\left(u, y_{n}\right) \\
\leq & \alpha_{n} d^{2}(u, p)+\left(1-\alpha_{n}\right)\left(\beta_{n} d^{2}\left(x_{n}, p\right)+\left(1-\beta_{n}\right) d^{2}\left(T_{n} z_{n}, p\right)\right) \\
& -\alpha_{n}\left(1-\alpha_{n}\right) d^{2}\left(u, y_{n}\right) \\
\leq & \alpha_{n} d^{2}(u, p)+\left(1-\alpha_{n}\right)\left(\beta_{n} d^{2}\left(x_{n}, p\right)+\left(1-\beta_{n}\right) d^{2}\left(z_{n}, p\right)\right) \\
& -\alpha_{n}\left(1-\alpha_{n}\right) d^{2}\left(u, y_{n}\right) \\
\leq & \alpha_{n} d^{2}(u, p)+\left(1-\alpha_{n}\right)\left(\beta_{n} d^{2}\left(x_{n}, p\right)+\left(1-\beta_{n}\right)\left(\gamma_{n} d^{2}\left(x_{n}, p\right)\right.\right. \\
& \left.\left.+\left(1-\gamma_{n}\right) d^{2}\left(T_{n} x_{n}, p\right)\right)\right)-\alpha_{n}\left(1-\alpha_{n}\right) d^{2}\left(u, y_{n}\right) \\
\leq & \alpha_{n} d^{2}(u, p)+\left(1-\alpha_{n}\right)\left(\beta_{n} d^{2}\left(x_{n}, p\right)+\left(1-\beta_{n}\right) d^{2}\left(x_{n}, p\right)\right) \\
& -\alpha_{n}\left(1-\alpha_{n}\right) d^{2}\left(u, y_{n}\right) \\
= & \left(1-\alpha_{n}\right) d^{2}\left(x_{n}, p\right)+\alpha_{n}\left(d^{2}(u, p)-\left(1-\alpha_{n}\right) d^{2}\left(u, y_{n}\right)\right),
\end{aligned}
$$


which implies

$$
d^{2}\left(x_{n+1}, p\right) \leq\left(1-\alpha_{n}\right) d^{2}\left(x_{n}, p\right)+\alpha_{n}\left(d^{2}(u, p)-\left(1-\alpha_{n}\right) d^{2}\left(u, y_{n}\right)\right) .
$$

Hence, by $A_{1}$ and Lemma 2.4, it is enough to show

$$
\limsup _{n}\left(d^{2}(u, p)-\left(1-\alpha_{n}\right) d^{2}\left(u, y_{n}\right)\right) \leq 0 .
$$

For showing this, we have

$$
\begin{aligned}
& d\left(y_{n}, x_{n}\right)=d\left(\beta_{n} x_{n} \oplus\left(1-\beta_{n}\right) T_{n} z_{n}, x_{n}\right) \\
= & \left(1-\beta_{n}\right) d\left(T_{n} z_{n}, x_{n}\right) \\
\leq & \left(1-\beta_{n}\right) d\left(T_{n} z_{n}, x_{n+1}\right)+\left(1-\beta_{n}\right) d\left(x_{n+1}, x_{n}\right) \\
\leq & \left(1-\beta_{n}\right)\left(\alpha_{n} d\left(u, T_{n} z_{n}\right)+\left(1-\alpha_{n}\right) d\left(T_{n} z_{n}, y_{n}\right)\right)+\left(1-\beta_{n}\right) d\left(x_{n+1}, x_{n}\right) \\
= & \left(1-\beta_{n}\right) \alpha_{n} d\left(u, T_{n} z_{n}\right)+\left(1-\beta_{n}\right)\left(1-\alpha_{n}\right) \beta_{n} d\left(T_{n} z_{n}, x_{n}\right)+\left(1-\beta_{n}\right) d\left(x_{n+1}, x_{n}\right)
\end{aligned}
$$

which follows

$$
d\left(y_{n}, x_{n}\right) \rightarrow 0 .
$$

On the other hand, by (3.6) and Lemma 2.6, we get

$$
\mu_{n}\left(d^{2}(u, p)-d^{2}\left(u, x_{n}\right)\right) \leq 0,
$$

for all Banach limits $\mu$. Thus

$$
\underset{n}{\limsup }\left(\left(d^{2}(u, p)-d^{2}\left(u, x_{n+1}\right)\right)-\left(\left(d^{2}(u, p)-d^{2}\left(u, x_{n}\right)\right)\right)=0 .\right.
$$

Therefore, by Lemma 2.3, we obtain

$$
\limsup _{n}\left(d^{2}(u, p)-d^{2}\left(u, x_{n}\right)\right) \leq 0 .
$$

Hence, by (3.7) and $\alpha_{n} \rightarrow 0$, we get

$$
\limsup _{n}\left(d^{2}(u, p)-\left(1-\alpha_{n}\right) d^{2}\left(u, y_{n}\right)\right)=\limsup _{n}\left(d^{2}(u, p)-d^{2}\left(u, x_{n}\right)\right) \leq 0 .
$$

That is the desired result.

The following corollary extends Theorem 2.1 of [15] as well as Theorem 3.1 of [6] and Theorem 2.3 of Saejung [17].

Corollary 3.2. Let $C$ be a closed and convex subset of complete $C A T(0)$ space $X$ and let $T: C \longrightarrow C$ be a nonexpansive mapping such that $F(T) \neq \emptyset$. Given a point $u \in C$ and the initial guess $x_{0} \in C$ is chosen arbitrarily. Suppose $\left\{x_{n}\right\}$ 
is generated by

$$
\left\{\begin{array}{l}
z_{n}=\gamma_{n} x_{n} \oplus\left(1-\gamma_{n}\right) T x_{n} \\
y_{n}=\beta_{n} x_{n} \oplus\left(1-\beta_{n}\right) T z_{n} \\
x_{n+1}=\alpha_{n} u \oplus\left(1-\alpha_{n}\right) y_{n}
\end{array}\right.
$$

where sequences $\left\{\alpha_{n}\right\},\left\{\beta_{n}\right\}$ and $\left\{\gamma_{n}\right\}$ in $[0,1]$, satisfy the following conditions: $\left(A_{1}\right) \sum_{n=1}^{\infty} \alpha_{n}=\infty$;

$\left(A_{2}\right) \alpha_{n} \rightarrow 0, \beta_{n} \rightarrow 0, \liminf _{n} \gamma_{n}>0$

$\left(A_{3}\right) \sum_{n=1}^{\infty}\left|\alpha_{n+1}-\alpha_{n}\right|<\infty$ or $\lim _{n \rightarrow \infty} \frac{\alpha_{n}}{\alpha_{n+1}}=1$;

$\left(A_{4}\right) \sum_{n=1}^{\infty}\left|\beta_{n+1}-\beta_{n}\right|<\infty$ or $\lim _{n \rightarrow \infty} \frac{\left|\beta_{n+1}-\beta_{n}\right|}{\alpha_{n+1}}=0$;

$\left(A_{5}\right) \sum_{n=1}^{\infty}\left|\gamma_{n+1}-\gamma_{n}\right|<\infty$ or $\lim _{n \rightarrow \infty} \frac{\left|\gamma_{n+1}-\gamma_{n}\right|}{\alpha_{n+1}}=0$.

Then $\left\{x_{n}\right\}$ converges strongly to a fixed point of $T$, which is the nearest point of $F(T)$ to $u$.

In the following examples, we show the parallel conditions on $\left\{\alpha_{n}\right\},\left\{\beta_{n}\right\}$ and $\left\{\gamma_{n}\right\}$, respectively, in $A_{3}, A_{4}$ and $A_{5}$ are independent.

Example 3.3. Let $k \geq 1$ be a positive integer. If the sequence $\left\{\alpha_{n}\right\}$ is given by

$$
\alpha_{n}=0 \quad \text { for } n=k^{2} \quad \text { and } \quad \alpha_{n}=\frac{1}{k^{2}} \quad \text { for } \quad(k-1)^{2}<n<k^{2}
$$

then $\alpha_{n} \rightarrow 0, \sum_{n=1}^{\infty} \alpha_{n}=\infty, \sum_{n=1}^{\infty}\left|\alpha_{n+1}-\alpha_{n}\right|<\infty$. But $\lim _{n \rightarrow \infty} \frac{\alpha_{n}}{\alpha_{n+1}}$ doesn't exist.

Example 3.4. Let $k \geq 0$ be an integer. If the sequences $\left\{\alpha_{n}\right\},\left\{\beta_{n}\right\}$ and $\left\{\gamma_{n}\right\}$ are given by

$\alpha_{n}=\frac{1}{\sqrt{n+1}}, \beta_{n}=\frac{1}{k+1}$, for $2^{k} \leq n<2^{k+1}, \gamma_{n}=\frac{k}{k+1}$ for $2^{k} \leq n<2^{k+1}$,

then $\alpha_{n} \rightarrow 0, \quad \sum_{n=1}^{\infty} \alpha_{n}=\infty, \quad \sum_{n=1}^{\infty}\left|\alpha_{n+1}-\alpha_{n}\right|<\infty, \quad \lim _{n \rightarrow \infty} \frac{\alpha_{n}}{\alpha_{n+1}}=1$, $\beta_{n} \rightarrow 0, \sum_{n=1}^{\infty}\left|\beta_{n+1}-\beta_{n}\right|<\infty, \lim _{n \rightarrow \infty} \gamma_{n}=1>0$ and $\sum_{n=1}^{\infty}\left|\gamma_{n+1}-\gamma_{n}\right|<$ $\infty$. But $\lim _{n \rightarrow \infty} \frac{\left|\beta_{n+1}-\beta_{n}\right|}{\alpha_{n+1}}=\infty$ and $\lim _{n \rightarrow \infty} \frac{\left|\gamma_{n+1}-\gamma_{n}\right|}{\alpha_{n+1}}$ does not exist.

Example 3.5. If the sequences $\left\{\alpha_{n}\right\},\left\{\beta_{n}\right\}$ and $\left\{\gamma_{n}\right\}$ are given by $\alpha_{n}=\frac{1}{\sqrt{n+1}} \quad$ and $\quad \beta_{2 n}=\frac{1}{\sqrt{n+1}}, \quad \beta_{2 n+1}=\frac{1}{\sqrt{n+1}}+\frac{1}{n+1}$

and $\gamma_{2 n}=1-\frac{1}{n+1}, \quad \gamma_{2 n+1}=1-\frac{2}{n+1}$.

Then $\alpha_{n} \rightarrow 0, \quad \sum_{n=1}^{\infty} \alpha_{n}=\infty, \quad \sum_{n=1}^{\infty}\left|\alpha_{n+1}-\alpha_{n}\right|<\infty, \quad \lim _{n \rightarrow \infty} \frac{\alpha_{n}}{\alpha_{n+1}}=1$, $\beta_{n} \rightarrow 0, \quad \lim _{n \rightarrow \infty} \frac{\left|\beta_{n+1}-\beta_{n}\right|}{\alpha_{n+1}}=0, \quad \gamma_{n} \rightarrow 1$ and $\lim _{n \rightarrow \infty} \frac{\left|\gamma_{n+1}-\gamma_{n}\right|}{\alpha_{n+1}}=0$. But $\sum_{n=1}^{\infty}\left|\beta_{n+1}-\beta_{n}\right|=\infty$ and $\sum_{n=1}^{\infty}\left|\gamma_{n+1}-\gamma_{n}\right|=\infty$. 
Example 3.6. Suppose that $\left\{\alpha_{n}\right\}$ is a sequence such that $\alpha_{n} \rightarrow 0$ and $\sum_{n=1}^{\infty} \alpha_{n}=\infty$. By setting $\beta_{n}=\alpha_{n}$ and $\gamma_{n}=1-\alpha_{n} \quad$ for each $n$, we have (i) $\beta_{n} \rightarrow 0$ and $\gamma_{n} \rightarrow 1$,

(ii) If $\sum_{n=1}^{\infty}\left|\alpha_{n+1}-\alpha_{n}\right|<\infty$, then $\sum_{n=1}^{\infty}\left|\beta_{n+1}-\beta_{n}\right|<\infty$ and $\sum_{n=1}^{\infty} \mid \gamma_{n+1}-$ $\gamma_{n} \mid<\infty$.

(iii) If $\lim _{n \rightarrow \infty} \frac{\alpha_{n}}{\alpha_{n+1}}=1$, then $\lim _{n \rightarrow \infty} \frac{\left|\beta_{n+1}-\beta_{n}\right|}{\alpha_{n+1}}=0 \quad$ and $\lim _{n \rightarrow \infty} \frac{\left|\gamma_{n+1}-\gamma_{n}\right|}{\alpha_{n+1}}=0$.

In the following Corollary, with setting $T_{n} \equiv T$, we obtain an analogue of [23, Theorem 4.5] in $\mathrm{CAT}(0)$ spaces.

Corollary 3.7. Let $C$ be a closed and convex subset of complete $C A T(0)$ space $X$ and let $T: C \longrightarrow C$ be a nonexpansive mapping such that $F(T) \neq \emptyset$. Given a point $u \in C$ and the initial guess $x_{0} \in C$ is chosen arbitrarily. Assume that the sequences $\left\{a_{n}\right\},\left\{b_{n}\right\}$ and $\left\{c_{n}\right\}$ in $(0,1)$, satisfy in the following conditions: (i) $a_{n}+b_{n}+c_{n}=1, \quad \forall n \geq 1$,

(ii) $a_{n} \rightarrow 0, b_{n} \rightarrow 0$,

(iii) $\sum_{n=1}^{\infty} a_{n}=\infty$,

(iv) $\sum_{n=1}^{\infty}\left|a_{n+1}-a_{n}\right|<\infty$, or $\lim \frac{a_{n+1}}{a_{n}}=0$, (v) $\sum_{n=1}^{\infty}\left|b_{n+1}-b_{n}\right|<\infty$.

Then $\left\{x_{n}\right\}$ is defined by

$$
\begin{gathered}
x_{n+1}=a_{n} u \oplus\left(1-a_{n}\right) y_{n}, \\
y_{n}=\frac{b_{n}}{1-a_{n}} x_{n} \oplus \frac{c_{n}}{1-a_{n}} T_{n} x_{n},
\end{gathered}
$$

converges strongly to a fixed point of $T$ which is the nearest point of $F(T)$ to $u$.

Proof. The proof is an immediate consequence of Theorem 2.1, by setting $\alpha_{n}=a_{n}, \beta_{n}=\frac{b_{n}}{1-a_{n}}$ and $\gamma_{n} \equiv 1$.

\subsection{Inexact Halpern Iteration}

In this short section, we consider the strong convergence of Halpern iteration with errors in the setting of $\mathrm{CAT}(0)$ spaces.

Theorem 3.8. Let $C$ be a closed and convex subset of complete $C A T(0)$ space $X$ and let $T: C \longrightarrow C$ be a nonexpansive mapping with a nonempty fixed point set $F(T)$. Suppose that $u, x_{1} \in C$ are arbitrary chosen and $\left\{x_{n}\right\}$ is iteratively 
STRONG CONVERGENCE OF A COMPOSITE HALPERN TYPE ITERATION

generated by the following algorithm:

Step (1): Take $y_{n+1}=\alpha_{n} u \oplus\left(1-\alpha_{n}\right) T x_{n}, \quad \forall n \geq 1$.

Step(2): Choose $x_{n+1} \in C$ such that $d\left(x_{n+1}, y_{n+1}\right) \leq e_{n}$, where $\left\{e_{n}\right\}$ is a sequence of nonnegative real numbers.

Step(3): Return to step(1).

Suppose $\left\{z_{n}\right\}$ be a sequence that generated by exact (without error) Halpern iteration

$$
z_{n+1}=\alpha_{n} u \oplus\left(1-\alpha_{n}\right) T z_{n}, \quad \forall n \geq 1,
$$

where $z_{1}=x_{1} \in C$ and $\left\{\alpha_{n}\right\}$ is a sequence in $(0,1)$ such that

(1) $\lim _{n \rightarrow \infty} \alpha_{n}=0$,

(2) $\sum_{n=1}^{\infty} \alpha_{n}=\infty$,

(3) $\sum_{n=1}^{\infty}\left|\alpha_{n+1}-\alpha_{n}\right|<\infty$ or $\lim _{n \rightarrow \infty} \frac{\alpha_{n}}{\alpha_{n+1}}=1$,

(4) $\sum_{n=1}^{\infty} e_{n}<\infty$ or $\lim _{n \rightarrow \infty} \frac{e_{n}}{\alpha_{n}}=0$.

Then $\left\{x_{n}\right\}$ converges to $z \in F(T)$, which is the nearest point of $F(T)$ to $u$.

Proof. By [17, Theorem 2.3], $\left\{z_{n}\right\}$ converges to $z \in F(T)$ which is the nearest point of $F(T)$ to $u$. On the other hand,

$$
\begin{aligned}
d\left(z_{n+1}, x_{n+1}\right) & \leq d\left(z_{n+1}, y_{n+1}\right)+d\left(y_{n+1}, x_{n+1}\right) \\
& \leq\left(1-\alpha_{n}\right) d\left(T z_{n}, T x_{n}\right)+e_{n} \\
& \leq\left(1-\alpha_{n}\right) d\left(z_{n}, x_{n}\right)+e_{n}
\end{aligned}
$$

Thus, by the conditions (2), (4) and Lemma 2.4, we get

$$
\lim _{n \rightarrow \infty} d\left(z_{n}, x_{n}\right)=0 .
$$

On the other hand,

$$
d\left(x_{n}, z\right) \leq d\left(x_{n}, z_{n}\right)+d\left(z_{n}, z\right)
$$

which together with $z_{n} \rightarrow z$ and (3.9) imply $x_{n}$ converges to $z \in F(T)$, which is the nearest point of $F(T)$ to $u$.

Remark 3.9. Theorem 3.8 improves the sumability assumption on the error sequence $\left\{e_{n}\right\}$ even in Banach spaces. For example, $e_{n}=\frac{1}{n}$ and $\alpha_{n}=\frac{1}{\sqrt{n}}$ satisfy the assumptions of Theorem 3.8 , but $\left\{e_{n}\right\}$ is not sumable.

\section{Acknowledgments:}

The author is grateful to the referees for their valuable comments and suggestions. 
STRONG CONVERGENCE OF A COMPOSITE HALPERN TYPE ITERATION

\section{References}

[1] Aoyama, K., Kimura, Y., Takahashi, W., Toyoda, M.: Approximation of common fixed points of a countable family of nonexpansive mappings in a Banach space. Nonlinear Anal. 67, (2007) 2350-2360.

[2] Bridson, M., Haefliger, A.: Metric Spaces of Non-Positive Curvature. Fundamental Principles of Mathematical Sciences. Springer, Berlin 319, (1999).

[3] Brown, K. S.: Buildings. Springer, New York, (1989).

[4] Chaoha, P., Phon-on, A.: A note on fixed point sets in CAT(0) spaces. J. Math. Anal. Appl. 320(2), (2006) 983-987.

[5] Chidume, C. E., Chidume, C. O.: Iterative approximation of fixed points of nonexpansive mappings. J. Math. Anal. Appl. 318, (2006) 288-295.

[6] Cuntavepanit, A., Panyanak, B.: Strong Convergence of Modified Halpern Iterations in CAT(0) Spaces. Fixed Point Theory Appl. 869458, (2011) doi:10.1155/2011/869458.

[7] Dhompongsa, S., Panyanak, B.: On $\Delta$-convergence theorems in $\operatorname{CAT}(0)$ spaces. Comput. Math. Appl. 56(10), (2008) 2572-2579.

[8] Goebel, K., Reich, S,: Uniform Convexity, hyperbolic Geometry, and nonexpansive Mappings. Monographs and Textbooks in Pure and Applied Mathematics, Marcel Dekker, Inc, New York, 83, (1984).

[9] Halpern, B.: Fixed points of nonexpanding maps. Bull. Amer. Math. Soc. 73, (1967) 957-961.

[10] Kim, T. H., Xu, H. K.: Strong convergence of modified Mann iterations. J. Math. Anal. Appl. 61, (2005) 51-60.

[11] Kirk, W. A.: Fixed point theorems in $\operatorname{CAT}(0)$ spaces and $\mathbb{R}$-trees. Fixed Point Theory Appl. 4, (2004) 309-316.

[12] Kirk, W. A.: Geodesic geometry and fixed point theory II. Paper presented at the International Conference on Fixed Point Theory and Applications, Yokohama Publ. (2004) 113-142 .

[13] Kirk, W. A.: Geodesic geometry and fixed point theory. Paper presented at the Seminar of Mathematical Analysis, University of Sevilla Secretry, sevilla, spain, 64, (2003) 195-225. 
STRONG CONVERGENCE OF A COMPOSITE HALPERN TYPE ITERATION

[14] Lions, P. L.: Approximation de points fixes de contractions. C.R. Acad Sci Paris Ser. A-B 284, (1977) A1357-A1359.

[15] Qin, X., Su. Y., Shang, M.: Strong convergence of the composite Halpern iteration. J. Math. Anal. Appl. 339, (2008) 996-1002.

[16] Reich, S.: Approximating fixed points of non-expansive mappings. Panam. Math. J. 4(2), (1994) 23-28.

[17] Saejung, S.: Halperns iteration in CAT(0) spaces. Fixed Point Theory Appl. 471781, (2010).

[18] Shioji, N., Takahashi, W.: Strong convergence of approximated sequences for non-expansive mappings in Banach spaces. Proc. Amer. Math. Soc. 125, (1997) 3641-3645.

[19] Suzuki, T.: A sufficient and necessary condition for Halpern-type strong convergence to the fixed point of nonexpansive mapping. Proc. Amer. Math. Soc. 135, (2007) 99-106.

[20] Wittmann, R.: Approximation of fixed points of nonexpansive mappings. Arch Math. 58, (1992) 486-491.

[21] $\mathrm{Xu}, \mathrm{H} . \mathrm{K}$ : : Another control condition in an iterative method for nonexpansive mappings. Bull. Austral. Math. Soc. 65, (2002) 109-113.

[22] $\mathrm{Xu}, \mathrm{H}$. K.: Iterative algorithms for nonlinear operators. J. London Math. Soc. 66, (2002) 240-256.

Sajad Ranjbar,

Department of Mathematics,

College of Sciences,

Higher Education Center of Eghlid,

Eghlid, Iran.

E-mail: sranjbar74@yahoo.com, sranjbar@eghlid.ac.ir. 